\title{
Research Paper: The Effect Of Selective Neck Muscle Release Techniques On Clinical Indicators of Patients with Migraine Headache: A Randomized Controlled Trial
}

\section{Tahere Rezaeian ${ }^{1} \oplus$, *Zahra Mosallanezhad ${ }^{1,2} \oplus$, Mohammad Reza Nourbakhsh ${ }^{3}$, Mehdi Ahmadi ${ }^{1}$, Mohammad Rostami ${ }^{1}$, Mehdi Norouzi ${ }^{4}$}

1. Department of Physical Therapy, University of Social Welfare and Rehabilitation Sciences, Tehran, Iran

2. Research Center on Aging, University of Social Welfare and Rehabilitation Sciences, Tehran, Iran

3. Physical Therapy Department, Faculty of Rehabilitation, University of North Georgia, The USA.

4. Social Determinants of Health Research Center, University of Social Welfare and Rehabilitation Sciences, Tehran, Iran

\begin{tabular}{|c|c|}
\hline $\begin{array}{l}\text { Use your device to scan } \\
\text { and read the article online }\end{array}$ & \\
\hline 回 & Cftation Rezaeian T, Mosallanezhad Z, Nourbakhsh MR, Ahmadi M, Rostami M, Norouzi N. [The Effect Of Selective Neck \\
\hline & $\begin{array}{l}\text { Muscle Release Techniques On Clinical Indicators of Patients with Migraine Headache: A Randomized Controlled Trial (Per- } \\
\text { sian)]. Archives of Rehabilitation. 2020; 20(4):384-397. http://dx.doi.org/10.32598/rj.20.4.384 }\end{array}$ \\
\hline 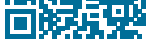 & doi ${ }^{8}$ http://dx.doi.org/10.32598/rj.20.4.384 \\
\hline
\end{tabular}

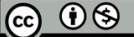

Received: 15 Apr 2019 Accepted: 05 Sep 2019 Available Online: 01 Jan 2020

Keywords:

Migraine headache, Myofascial Trigger Points, Manual Therapy, Neck muscles.

\section{ABSTRACT}

Objective Subjects with migraine headache often have stiff posture, especially in the head and neck. Also, these patients often have reduced cervical range of motion, faulty posture, forward head posture, increased sensitivity on the cervical muscles, and myofascial pain syndrome. Myofascial Trigger Points (MTrPs) are considered as the contributing factors to migraine headache. They produce painful trigger points in the cervical muscles. There are different techniques for eliminating MTrPs such as soft tissue (neck muscles) release technique, Ultrasound, and deep heat therapy, laser therapy, and dry needling. The current study aimed to investigate the effects of selective neck muscle release techniques on clinical indicators (frequency/intensity/duration of headache, medication, functional disability, and pressure pain threshold) in patients with migraine headache.

Materials \& Methods The current study is a randomized controlled trial was conducted in the physical therapy research center of Shiraz University of Medical Sciences in Iran from August 2017 to February 2018. The statistical population consisted of all patients with migraine headache. Of these, 40 patients (aged 25-55 years) with trigger points in neck muscles including sternocleidomastoid, upper trapezius, and suboccipital were selected and then randomly assigned into two groups of release (mean age, $40.4 \pm 11.2$ years) and control (mean age, $37.45 \pm 8.9$ years). The subjects in the release group received 6 sessions for 2 weeks (combined MTrP therapy and stretching movements), while control group received placebo superficial massage. Headache frequency/intensity/duration, medication, functional disability, and Pressure Pain Threshold (PPT) were assessed before, immediately after intervention, and at 1-month follow-up period. For data analysis, the two-way mixed design of ANOVA was used in SPSS v.23 software to evaluate the main effects of the two factors of group and time on the dependent variables. If the group effect was significant, independent t-test was performed to evaluate the differences in study variables between the two groups after the treatment and at the follow-up period, and if the time effect was significant, the paired t-test was carried out to assess the differences between the two groups before and after treatment and at the follow-up period. The significance was considered at $\mathrm{P}<0.05$

Results All the variables had normal distribution ( $P>0.05$ ); therefore, parametric tests were used for analyzing data. Based on the results, there were no significant differences between the two groups in all quantitative and qualitative variables before treatment $(P>0.05)$. The release group showed

\section{* Corresponding Author:}

Zahra Mosallanezhad, PhD.

Address: Department of Physical Therapy, University of Social Welfare and Rehabilitation Sciences, Tehran, Iran

Tel: +98 (21) 22180039

E-Mail: zmosallanezhad@yahoo.com 
improvement in the headache frequency, intensity, and duration, medication use, the functional ability, and the PPT level in comparison with the control group $(p<0.001)$. According to the paired t-test results, the release group showed a significant reduction in headache parameters, medication use and functional disability and increased PPT after the intervention and the follow-up period $(\mathrm{P}<0.001)$.

Conclusion The release techniques for selective neck muscles including MTrP therapy and stretching movements were helpful in improving some clinical indicators such as headache parameters, medication use, functional ability, and PPT in a short time. Therefore, these techniques can be recommended for treating migraine headache patients with MTrPs in sternocleidomastoid, upper trapezius, and suboccipital muscles. 
This Page Intentionally Left Blank 


\title{
تأثير تكنيكهاى آزادسازى عضلات منتخب تردن روى شاخصهاى بالينى در بيماران سردرد

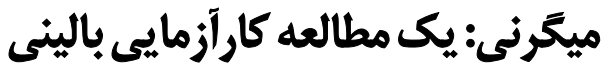

\author{
طاهره رضاييان' (ه، "زهرا مصلىنزادّاه، محمدرضا نوربخش"، مهدى احمدى'، محمد رستمى'، مهدى نوروزى"
}

1. ا.كروه فيزيوترايى، مانشكاه علوم بهزيستى و توانبخشى تهران، يراني

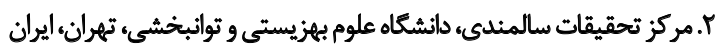

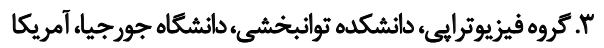

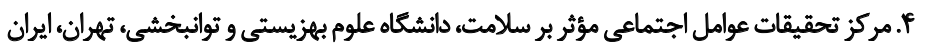

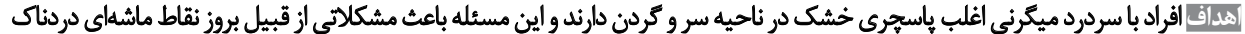

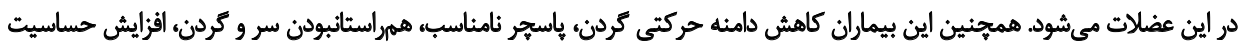

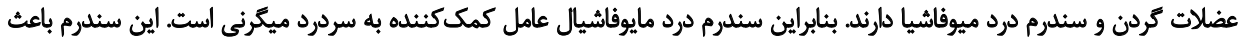

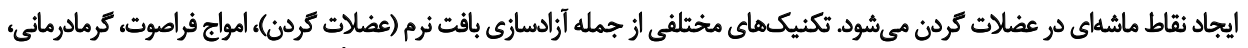

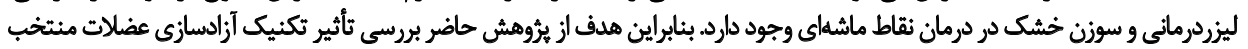

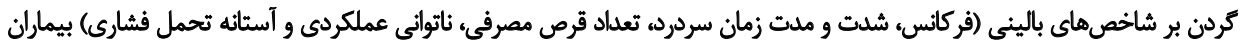

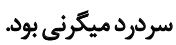

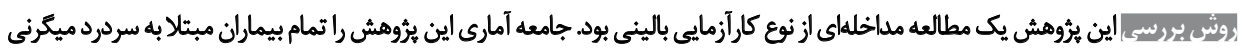

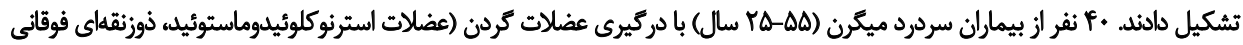

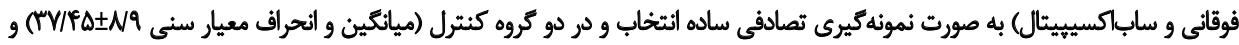

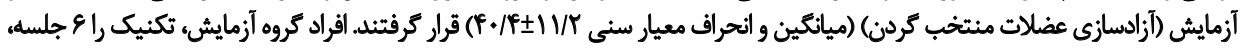

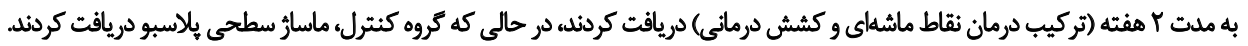

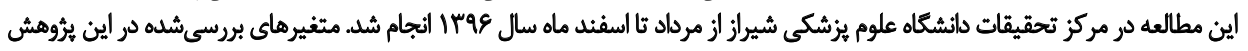

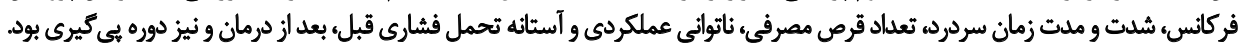

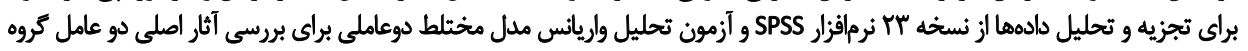

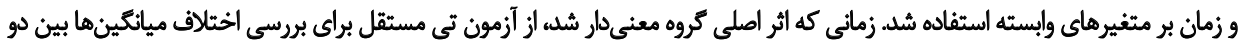

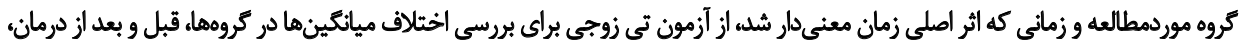

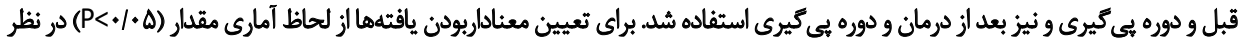
كرفته شد.

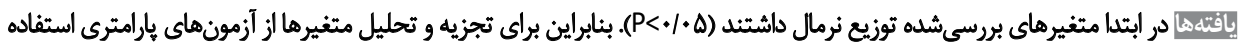

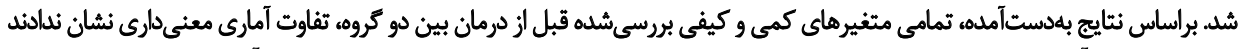

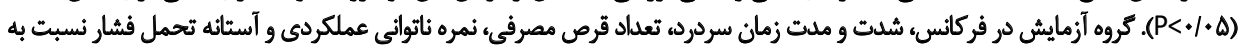

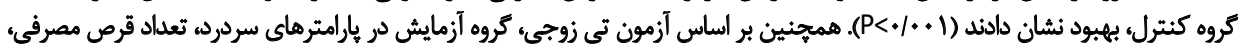

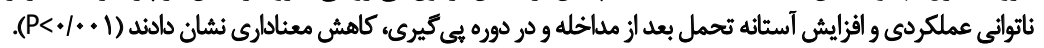

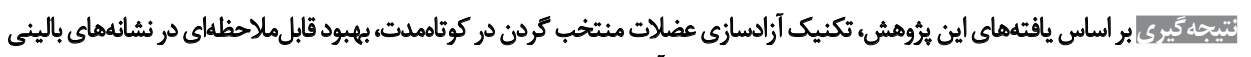

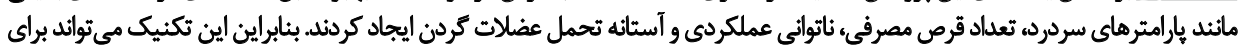

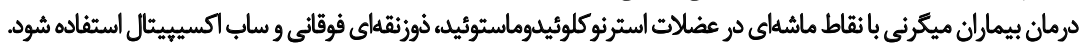

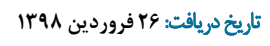

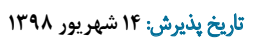

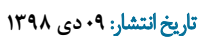

كليدواثرها:

اختلالات ميكرني، نقاط

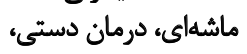
عضلات كردن 
دارودرمانى به دليل عوارض جانبى، براي همه بيماران مناسب

مقدمه

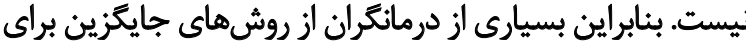

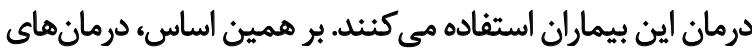

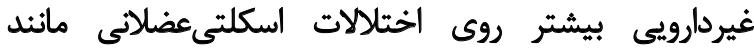

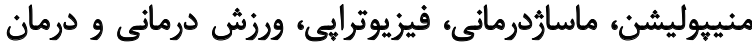

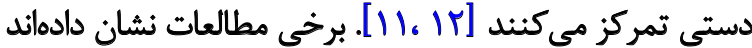

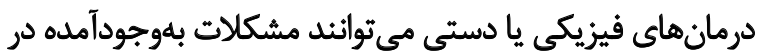

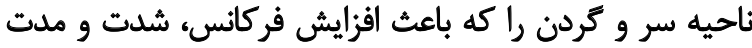

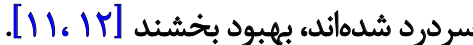
جان" و همكارانش مطالعهاى با هدف مقايسهكردن ثأثير

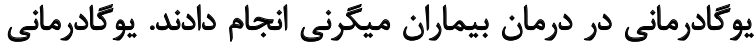

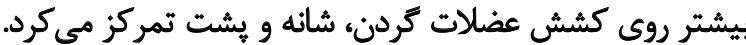

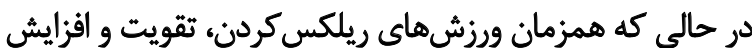

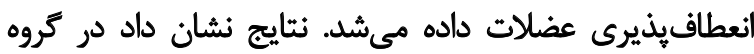

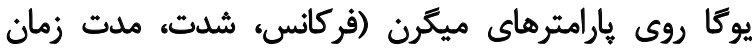

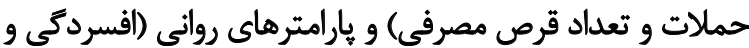

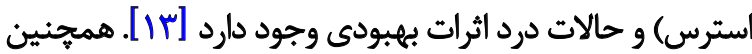

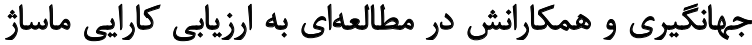

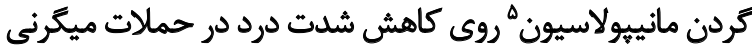

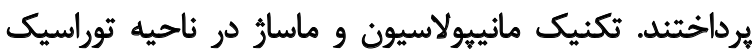

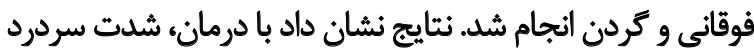

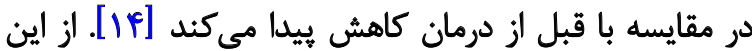

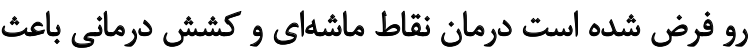
كاهش علاثم بالينى در اين بيماران مى نشود.

با توجه به شيوع تسترده سردرد ميكرنى و نياز اين بيماران

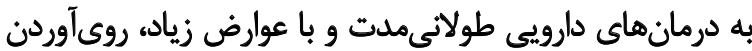

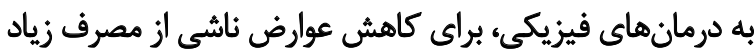

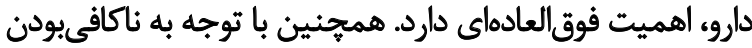

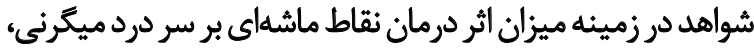

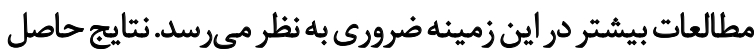

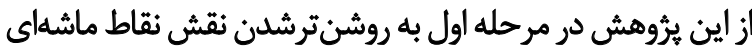

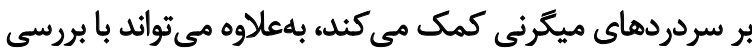

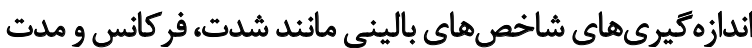

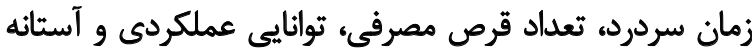

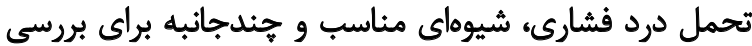

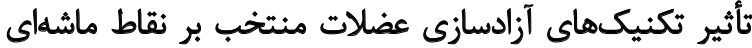
مدنظر رادر اختيار قرار دهد. ت ماندي

با بررسى هاى ما مشخص شد شواهد كافى درباره ميزان

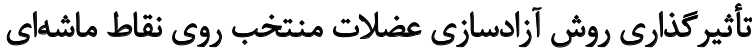

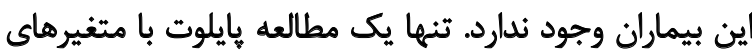

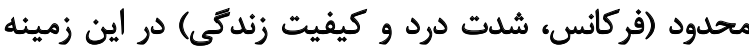

يكى از شايعترين مشكلات در زمينه يزشكى سردرد است

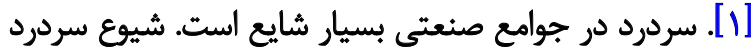

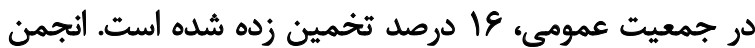

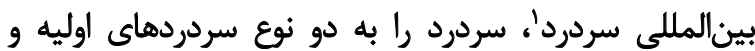

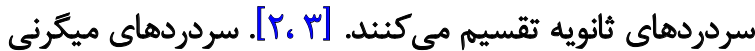

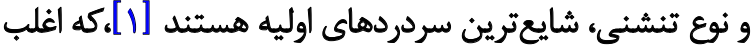

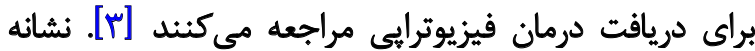

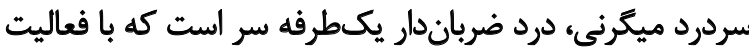

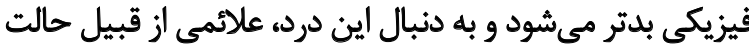

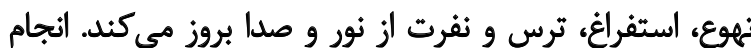

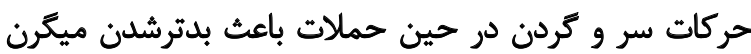

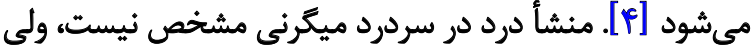

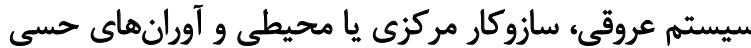

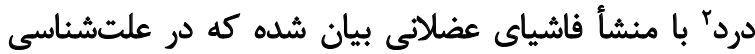

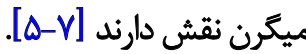

معاينات بالينى افراد مبتلا به ميكرن نشان داده است كه

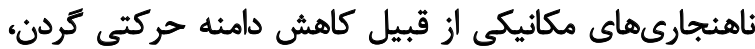

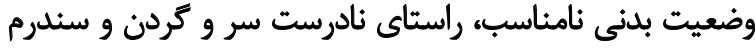

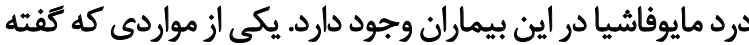

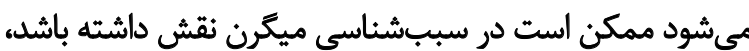

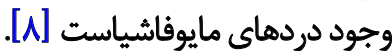

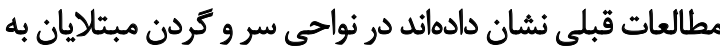

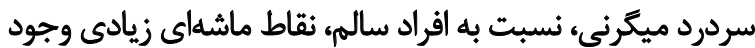

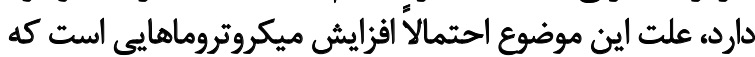

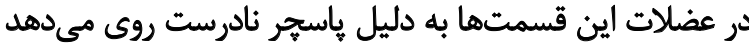

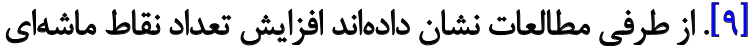

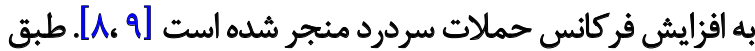

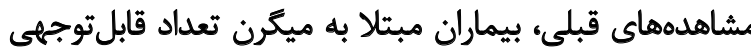

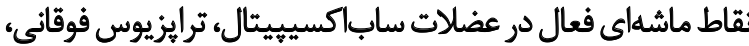

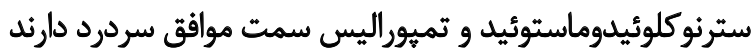

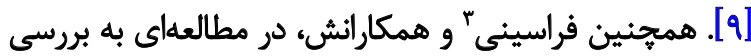

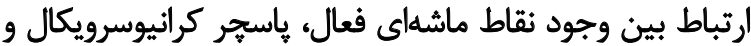

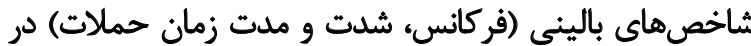

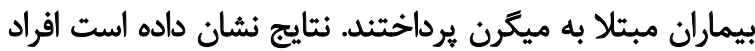
مبتلابه ميكرن نقاط ماشهاي فعال و ينهان در عضلات مطرحشده

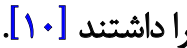
براى درمان بيماران مبتلا به سردرد ميكُرن، روشهاى درمانى

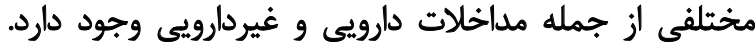

3. Ferracini 
همجينين افرادى كه هر كونه سابقه تروما در ناحيه كردن،

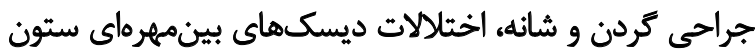

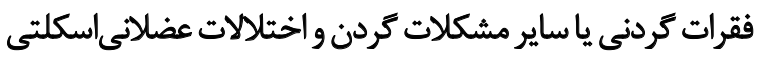

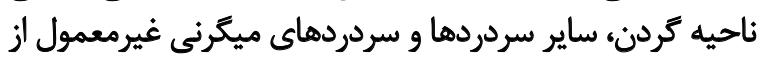

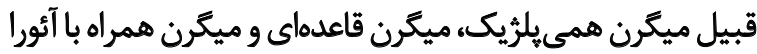

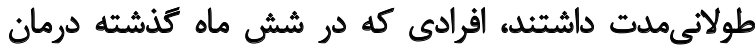

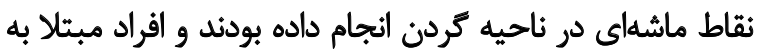

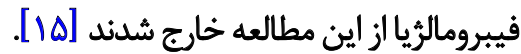
درمانكرى كه مداخله رالنجام داد، در ارتباط با رازيابى متغيرهاى

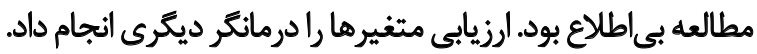

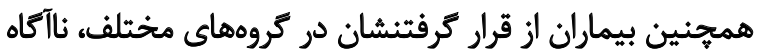

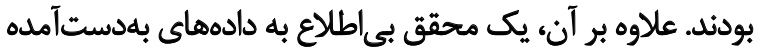

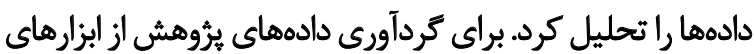

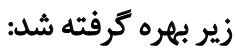

فرم يادداشث روزانه

براى جمع آورى اطلاعات از فرم يادداشت روزانه سردرد استفاده

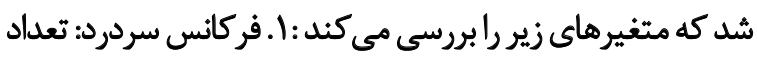

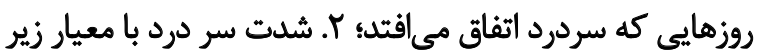
توسط بيمار ثبت مي كردود:

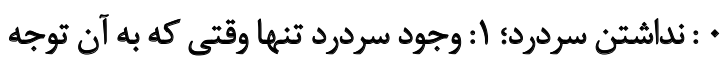

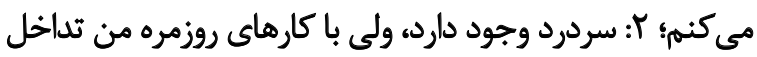

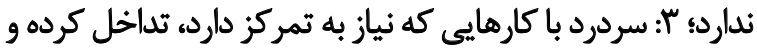

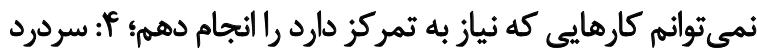

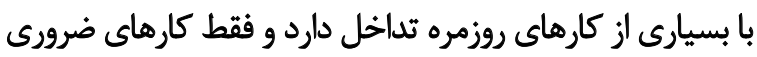

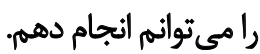

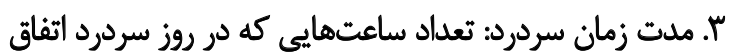

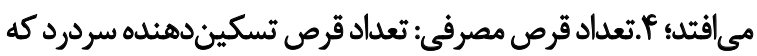

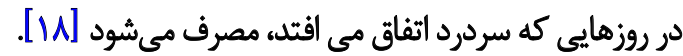

يوسش نامه سنجش ناتوانى عملكردى ناشيى أز سردرد

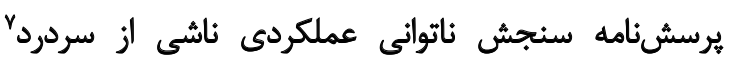

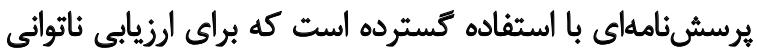

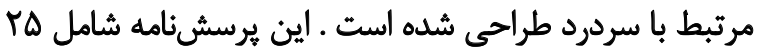

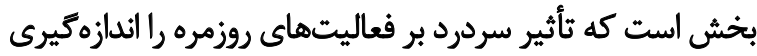

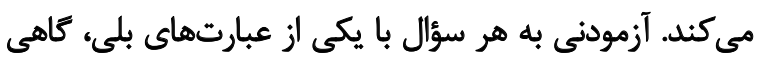

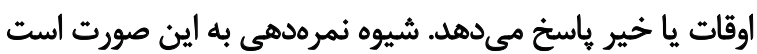

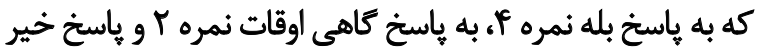

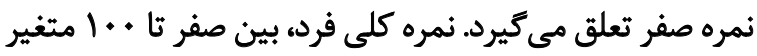

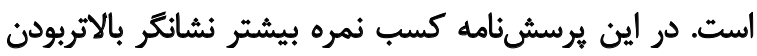

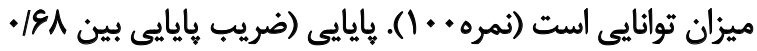

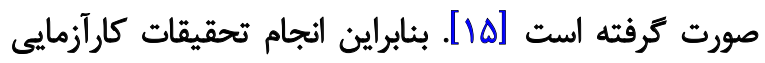

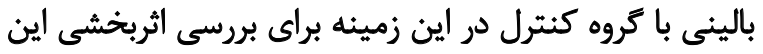

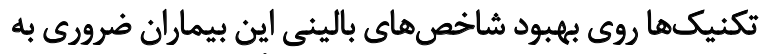

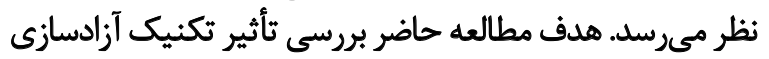

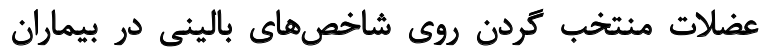

$$
\text { رودرد ميكرنى است. }
$$

يرُوهش حاضر يك مطالعه مداخلهاي از نوع كار آزمايى بالينى

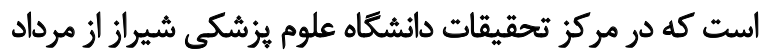

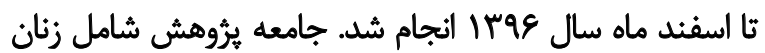

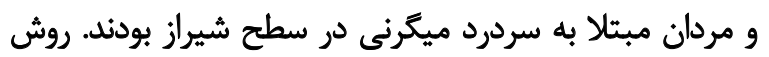

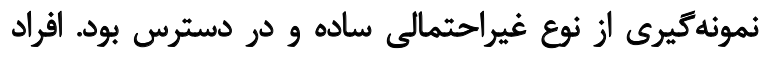

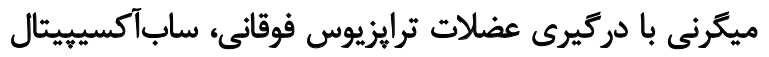

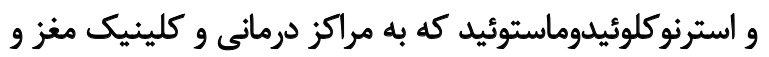

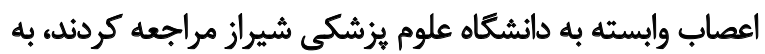

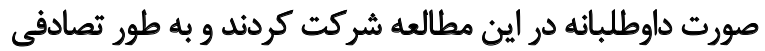

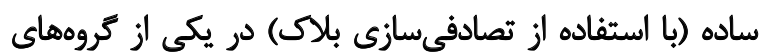

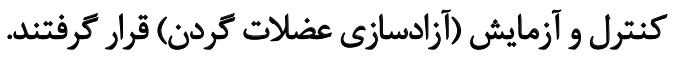

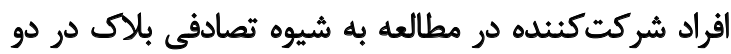

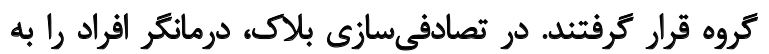

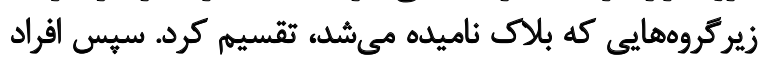

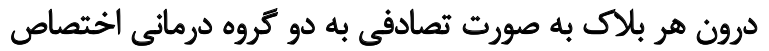

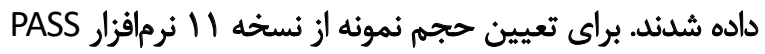

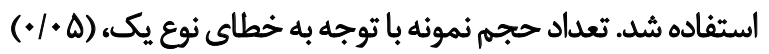

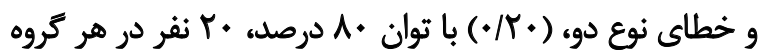

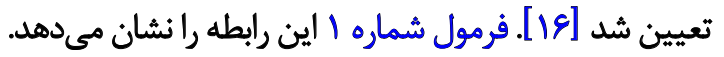

$$
n=\frac{\left(z_{1-\frac{a}{2}}+z_{1-\beta}\right)^{2}\left(\delta_{1}^{2}+\delta_{1}^{2}\right)}{\left(\mu_{1}-\mu_{2}\right)^{2}}=20
$$

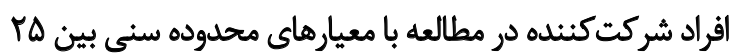

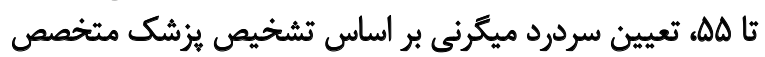

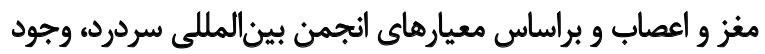

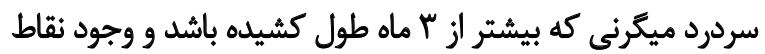

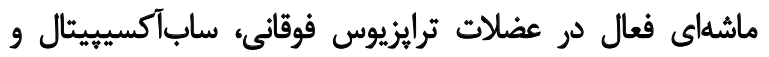

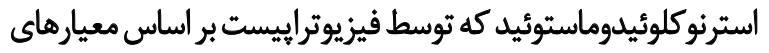

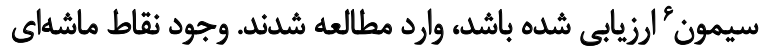

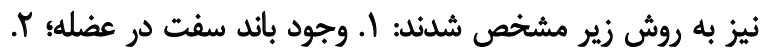

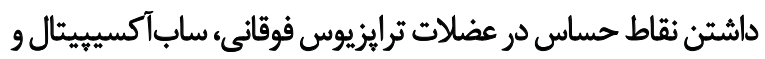

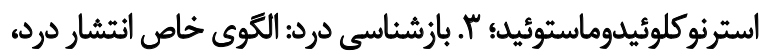

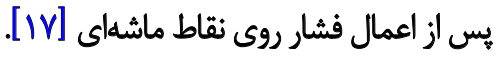




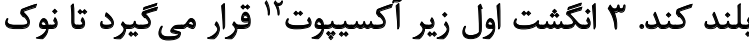

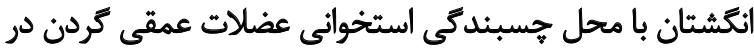

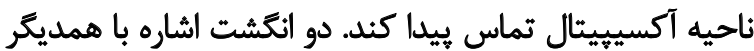

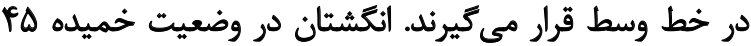

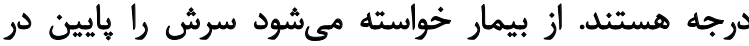

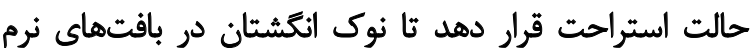

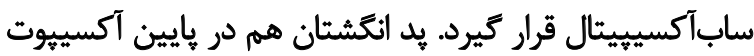

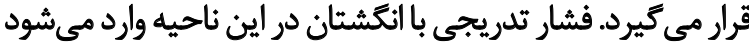

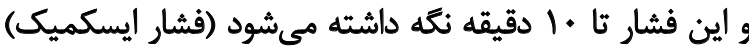

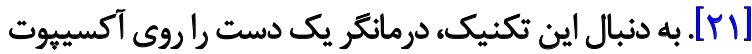

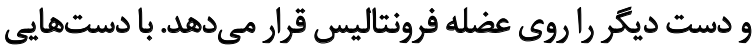

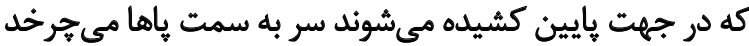

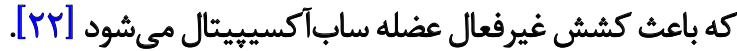

درمان نقاط ماشهاى عضله استرنوكلوئيدوماستوئيد و كشش آن

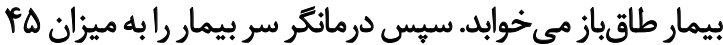

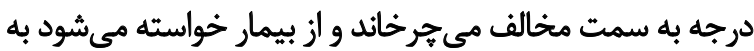

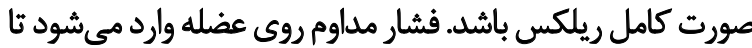

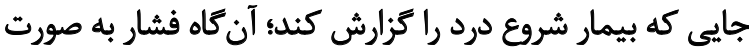

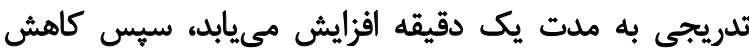

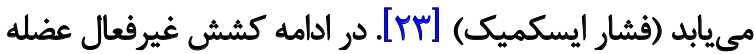

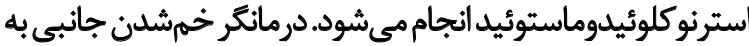

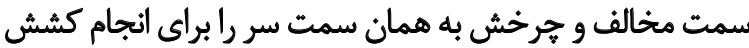

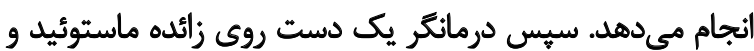

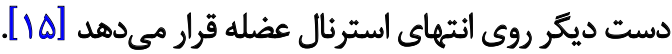

$$
\text { كروه كثترل (ماسار سطحى ثاسلاسب) }
$$

در اين گروه بيماران هيجزونه مداخلهاى دريافت نكردند.

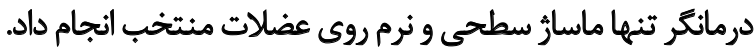

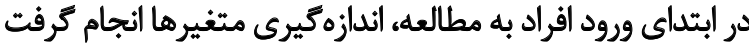

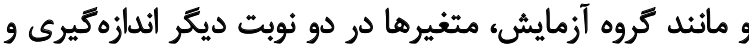

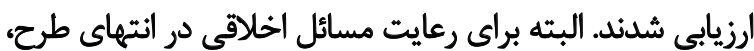

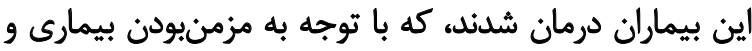

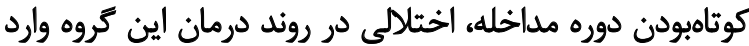

از بيماران خواسته شد هيج نوع داروى ضدالتهاب و شل كنينده

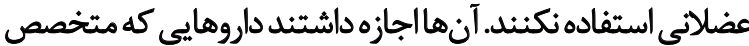

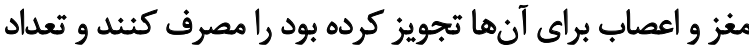

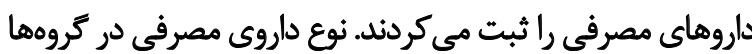

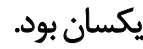

12. Occiput

13. Placebo superficial massage

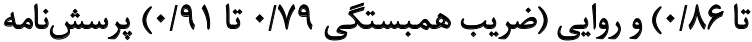
در بيماران ميكرنى فارسىزبان تأييد شده بود [19 آ]

أسثانه تحمل فُشارث

الكومتر فشارى براى كمّى كردن آستانه تحمل روى نقاط ماشهاى

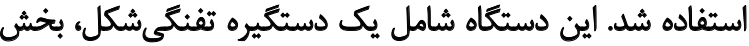

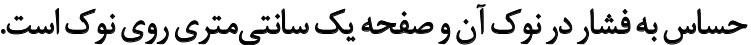

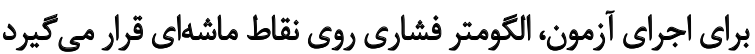

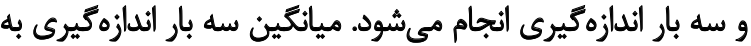

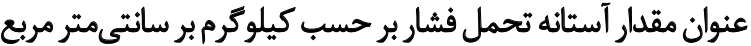

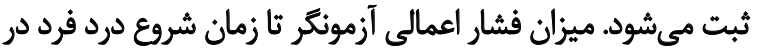

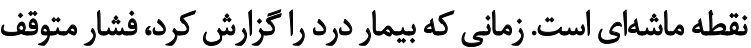

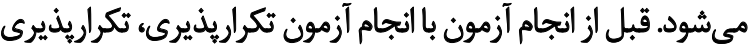

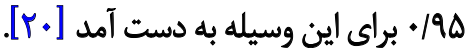

انجام مداخله به اين روش انجام شد: درمان شش جلسه در

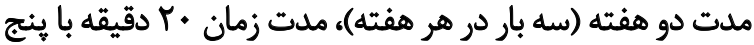

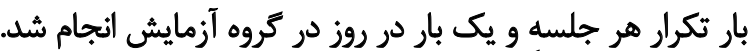

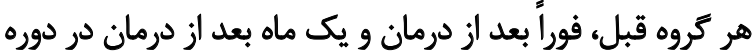

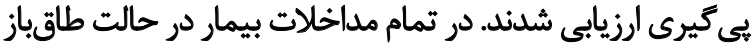

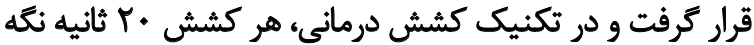
داشته مىشد. كو الز مايش

\section{درمان نقاط ماشهاى عضله ذوزنقهاى فوقانى و كشش آن}

بيمار طاقباز مي خوابد. سيس درمانكر سر بيمار را FA درجه

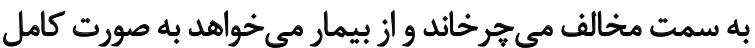

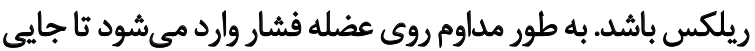

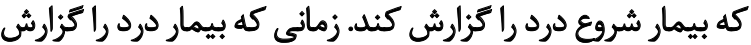

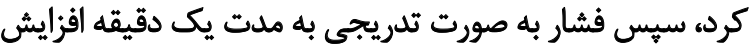

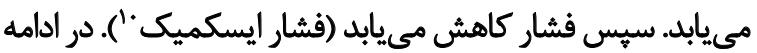

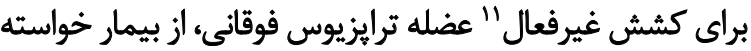

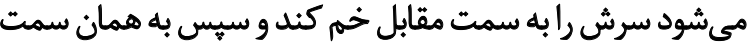

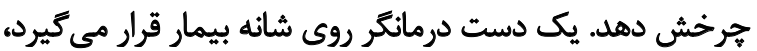

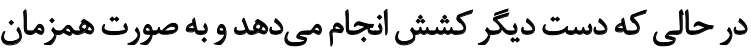

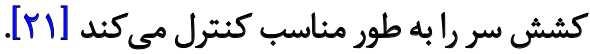

\section{مهار بافت نرم ساب آكسيييتال و كشش آن}

بيمار طاقباز مىخوابد. درمانتحر روى يك صندلى بالاى تخت

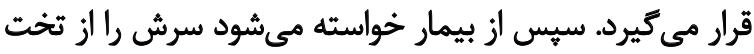

\section{Pressure Pain Threshold (PPT)}

9. Force Gauge, FG-5005, RS232, Leutron Electronic, Arizona, USA 10. Ischemic pressure

11. Passive stretch 
درمان و دوره بيى كيرى كاهش بيدا كرد. علاوه بر اين، ميانكين

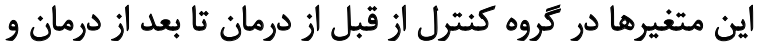

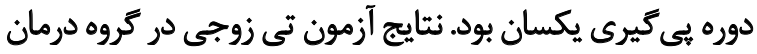

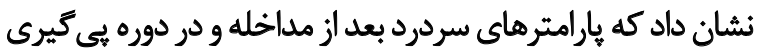

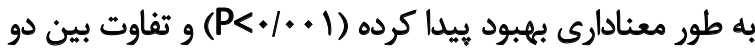

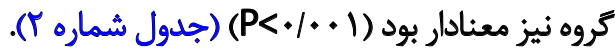

\section{شاخص ناثواني عملكردى مرثبط با سردرد}

در كروه آزمايش ميانكين كاهش نمره شاخص ناتوانى

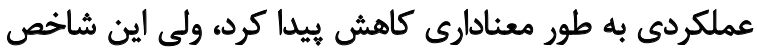

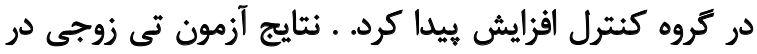

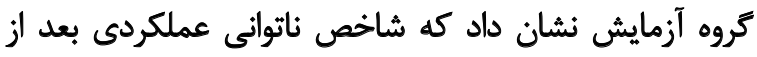

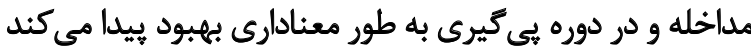

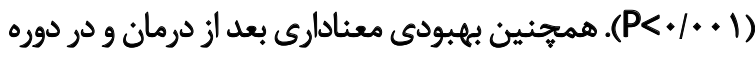

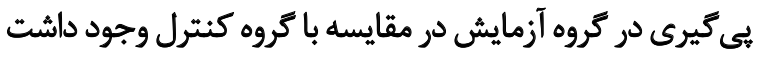

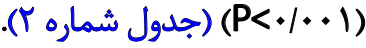

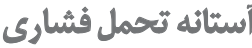

در نهايت، ميانكين نمرههاى آستانه تحمل فشارى عضلات

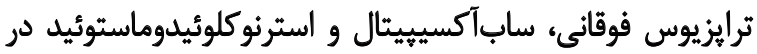

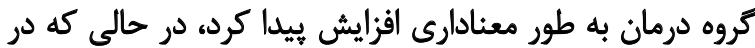

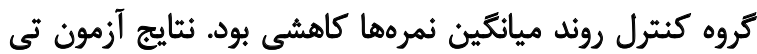

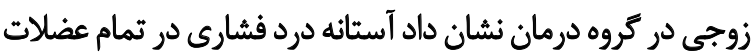

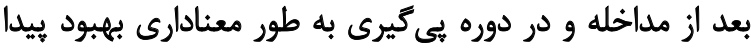

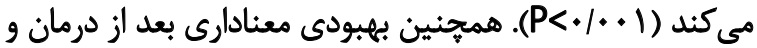

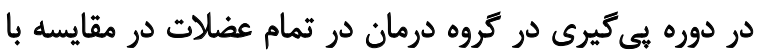

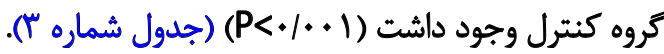

بحث

نتايج مطالعه حاضر نشان داد تكنيك آزادسازى عضلات تردن

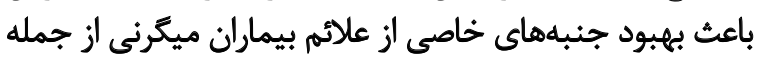

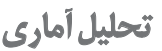

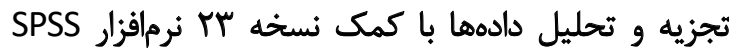

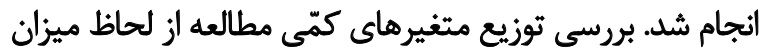

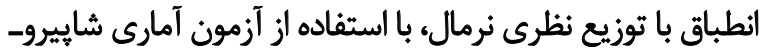

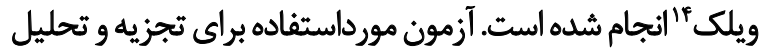

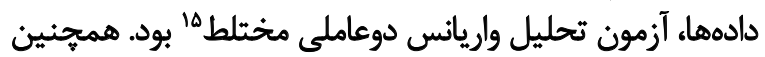

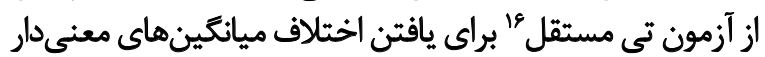

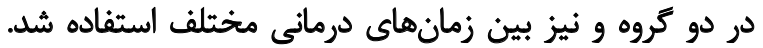

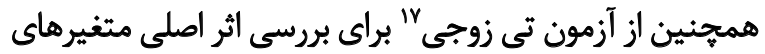

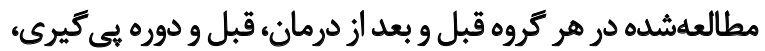

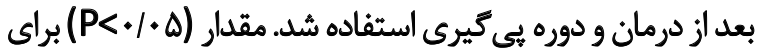

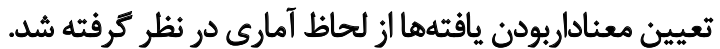

ياقتثلها

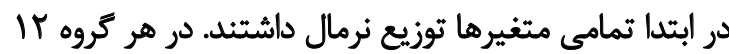

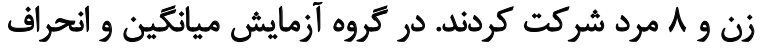

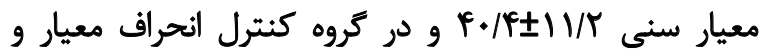

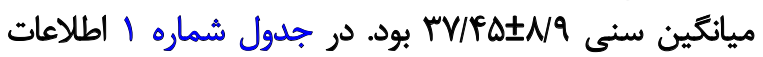

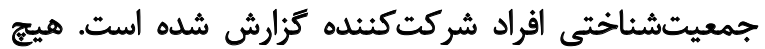

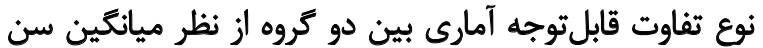

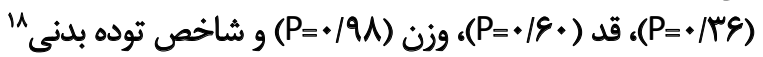

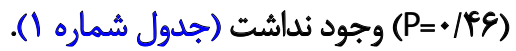
شاخُص هاي سردود در كروه آزمايش ميانكّين نمرههاى فركانس، شدت و مدات

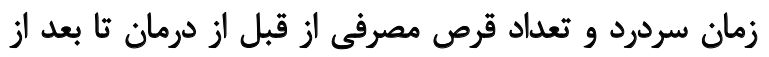

14. ShaPiro-Wilk

15. Two-way mixed design ANOVA

16. Independent t-test

17. Paired t-test

18. Body Mass Index (BMI)

جدول (ـاطلاعات جمعيتشُشاختى آزمودنىها (•r نفر)

\begin{tabular}{|c|c|c|c|}
\hline \multirow{2}{*}{ P } & \multicolumn{2}{|c|}{ مياتكين+انحراف معيار } & \multirow{2}{*}{ متغيرها } \\
\hline & تروه أزمايش & تروه كتترل & \\
\hline $\begin{array}{c}+/ 48 \\
t=* / 91\end{array}$ & $r \cdot R \cdot \pm 11 / T r$ & $T V / F \Delta \pm N G$ & سن (سال) \\
\hline $\begin{array}{c}. / u \\
t=* / . r\end{array}$ & V/\&N+1.9 & $\mathbb{W}+ \pm \cdot N$ & قد (سائتىمتر) \\
\hline$t=. / \Delta r$ & $\gamma+/ r+ \pm q / 10$ & $V+/ r \Delta \pm \& / V I$ & 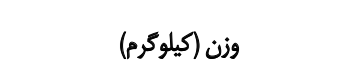 \\
\hline $\begin{array}{l}. / 48 \\
t=\bullet / M^{2}\end{array}$ & $r \Delta /=Y \pm r / \Psi$. & $r+/ T Y \pm T / \& g$ & شاخص توده بدنى (كيلوكرم بر متر مربع) \\
\hline
\end{tabular}

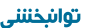


جدول T. مقايسه متغيرهاي فركائس، شدت و مدت سردرد و تعداد قرص مصرفي بين دو كروه كنترل و درمان در زمانهاي مختلف

\begin{tabular}{|c|c|c|c|c|c|c|c|c|c|c|}
\hline \multicolumn{2}{|c|}{$\mathrm{P}^{* * * *}$} & \multicolumn{2}{|c|}{ كروه آزمايش } & \multicolumn{2}{|c|}{ كروه كثترل } & \multirow{2}{*}{$\mathbf{P}^{*}$} & \multicolumn{2}{|c|}{ ميانكتين+|تحراف معيار } & \multirow{2}{*}{\multicolumn{2}{|c|}{ متغيرها }} \\
\hline ليّى كيرى & بعد & هيى قبل - & قبل-بعد & بيى قبلي - & قبل-بعد & & كروه أزمايش & كروه كتثرل & & \\
\hline $\begin{array}{l}<\cdot 1 \cdot \bullet 1 \\
\mathrm{t}=N \mid r\end{array}$ & $\begin{array}{l}<+1 .+1 \\
V / 80\end{array}$ & $\begin{array}{l}<+1 \cdot . \cdot 1 \\
\mathrm{t}=\mid r / \& 9\end{array}$ & $\begin{array}{l}<+1 \cdot+1 \\
\mathrm{t}=1 Y / A \Delta\end{array}$ & $\stackrel{\sim T A}{\mathrm{t}=* / 199}$ & $\stackrel{. / M}{t=1 / \Delta \Delta}$ & $<.1 .+1$ & $\begin{array}{l}r / A \Delta \pm 1 / M \\
V / T+ \pm \cdot / M \\
V / T+ \pm \cdot / R q\end{array}$ & $\begin{array}{l}r / \Delta \cdot \pm \cdot N E \\
r / Y \cdot \pm \cdot / A r \\
T / Y+ \pm \cdot / q Y\end{array}$ & يقيليل يقدى & فركانس \\
\hline $\begin{array}{l}<\cdot / *+1 \\
t=q / \cdot 1\end{array}$ & $\begin{array}{c}<+1++1 \\
\text { s/me }\end{array}$ & $\begin{array}{l}<\cdot / \cdot .1 \\
t=11 / \lambda_{*}\end{array}$ & $\begin{array}{l}<+1 \cdot .+1 \\
\mathrm{t}=1 \% / .9\end{array}$ & $\stackrel{. / T \Delta}{\mathrm{t}=+/ 9 t^{+}}$ & $\stackrel{* / f a}{t=* / V 8}$ & $<+1++1$ & $\begin{array}{l}r / 8 \Delta \pm 1 /+1 \\
V / A+ \pm+/ / 8 \\
1 / F \Delta \pm / / A\end{array}$ & $\begin{array}{l}r / r \Delta \pm=M \\
r / r+ \pm+/ g 1 \\
r / 1 \Delta \pm \cdot / R\end{array}$ & قبلى بعل & شدت سردرد \\
\hline $\begin{array}{l}<.1 . * 1 \\
\mathrm{t}=V / \Delta f^{\circ}\end{array}$ & $\begin{array}{l}.1 \cdot .1 \\
f / \pi \Delta\end{array}$ & $\begin{array}{l}* / .+1 \\
t=1 * 1 * .\end{array}$ & $\begin{array}{l}<+1 \cdot * 1 \\
\mathrm{t}=11 / \pi r\end{array}$ & $\stackrel{. / 48}{\mathrm{t}=+/ 9 t^{2}}$ & $\stackrel{. / T Y}{\mathrm{t}=1 / / r}$ & $<+1+\infty 1$ & 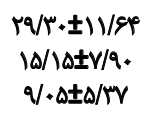 & 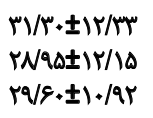 & قبلى بعدي & ملت زمران \\
\hline $\begin{array}{l}<\cdot 1 \cdot .+1 \\
\mathrm{t}=r \cdot \mid \& A\end{array}$ & $\begin{array}{l}<.1 . .1 \\
1+M V\end{array}$ & $\begin{array}{l}<\cdot 1 \cdot .01 \\
\mathrm{t}=r \mid / \& g\end{array}$ & $\begin{array}{l}<+1.01 \\
\mathrm{t}=\mid 9 / / 1\end{array}$ & $\stackrel{. / M A}{\mathrm{t}=\$ / T a}$ & $\begin{array}{l}.1 .9 \\
t=1 / 9 r\end{array}$ & $<* / * .1$ & 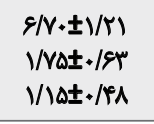 & $\begin{array}{l}8 / \Delta \cdot \pm 1 / 48 \\
\Delta / 9 \Delta \pm / / \cdot 9 \\
8 / 1 \Delta \pm / / \pi\end{array}$ & قبلى بعل & تعداد قرصى \\
\hline
\end{tabular}

توانبخننى

*P-value for independent t-test

**P-value for paired t-test in treatment group

*** $\mathrm{P}$-value for paired t-test in control group

**** $\mathrm{P}$-value for repeated measurement

تركيبى آزادسازى وضعيتى و دارو درمانى مؤثرتر از درمان دارويي

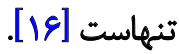

در مطالعه ديخرى قنبرى و همكاران به منظور مقايسه تأثير

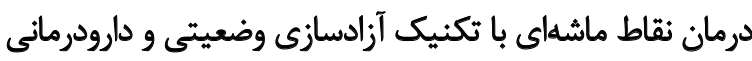

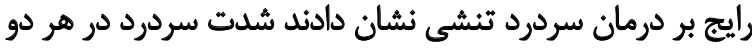

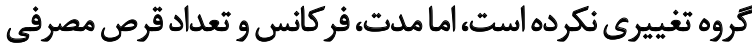

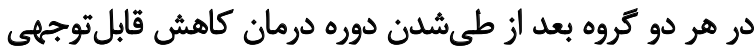

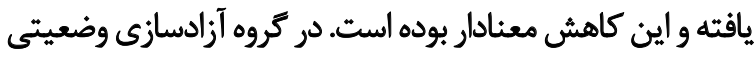

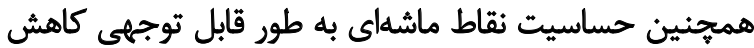

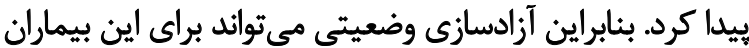

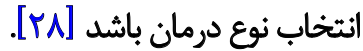

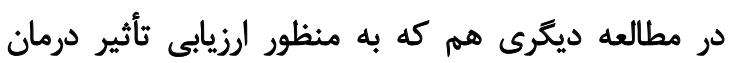

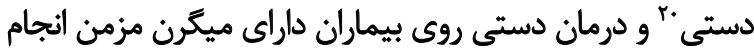

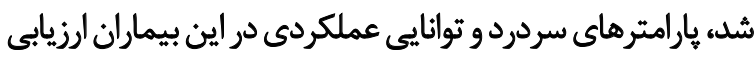

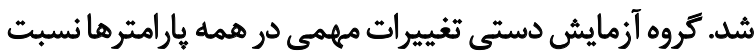

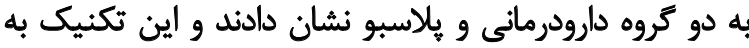

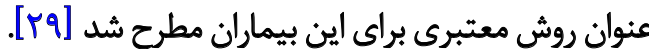

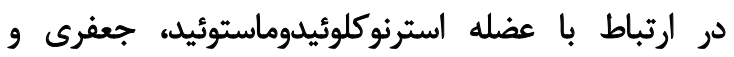

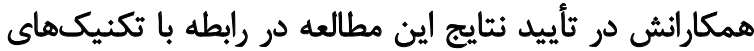

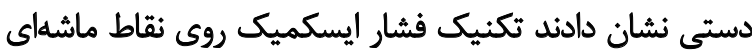

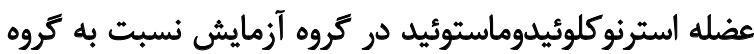

فركانس، شدت و مدت زمان سردرد، تعداد قرص مصرفى، ناتوانى

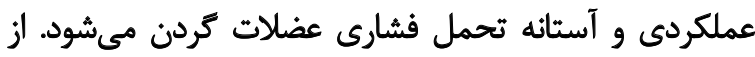

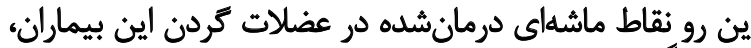

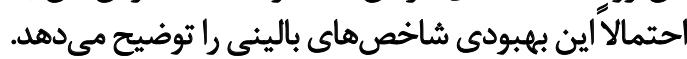

بر اساس يافتهماى اين مطالعه درمان درستى و آزادسازى

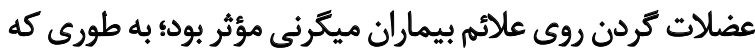

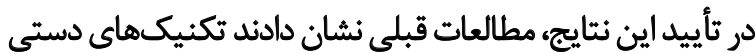

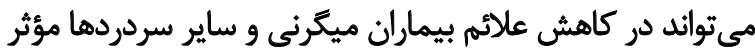

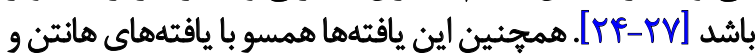

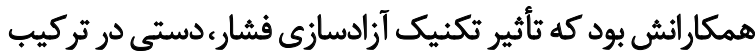

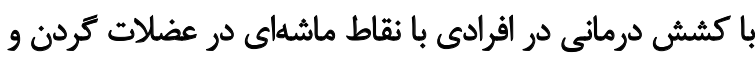

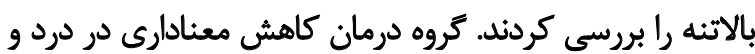

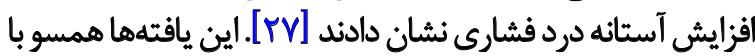

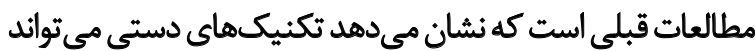

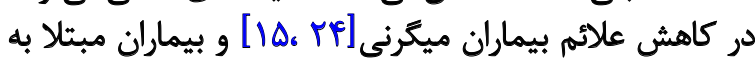

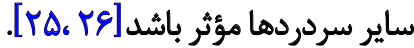
همجنين قنبرى و همكارانش همسو باين مطالعه، تأثير درمان

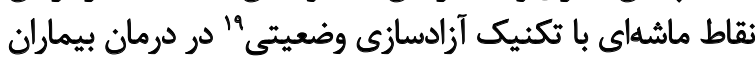

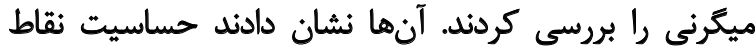
ماشهاى و دامنه حركتى تردن در تروني

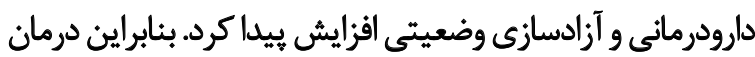

19. Positional release technique 
جدول با. مقايسه متغيرهاي ناتواني عملكردى، آستانه تحمل ذوزنقهاى فوقاني، آستائه تحمل سابآكسيييتال و آستائه تحمل استرنوكلوئيد و ماستوئيد بين دو كروه

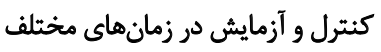

\begin{tabular}{|c|c|c|c|c|c|c|c|c|c|c|}
\hline \multicolumn{2}{|c|}{$\mathbf{P} * * * *$} & \multicolumn{2}{|c|}{ مروه آزمايش } & \multicolumn{2}{|c|}{ Fروه كثترل } & \multirow{2}{*}{ P* } & \multicolumn{2}{|c|}{ ميانكين+|نحراف معيار } & & \multirow{2}{*}{ مثغيرها } \\
\hline بيى كيرى & بعد & قيى قبلي & قبل-بعد & قيلى قيرى & قبل-بعد & & كروه آزمايش & مروه كثترل & & \\
\hline$<+1 \cdot+1$ & $<\cdot 1 \cdot+1$ & $<.1 .+1$ & $<.1 .+1$ & $<.1 . .1$ & .0 .0 & & $F \Delta / r+ \pm \mid F / F r$ & $r+/ 9+ \pm q / \pi r$ & قبل & \multirow{6}{*}{ 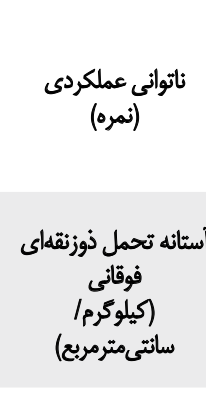 } \\
\hline \multirow[t]{2}{*}{$t=N \cdot A$} & $t \equiv t / 4$ & $t=11 / 8 \mathrm{~g}$ & $\mathrm{t}=\mathrm{N} / \mathrm{I}$ & $\mathrm{t}=\Delta / \mathrm{rA}$ & $t=r / / \Delta^{\circ}$ & $<.1 . .1$ & YNG.IVM & $M T / N \cdot \pm V E V$ & بعل & \\
\hline & & & & & & & $r V / * \pm N G$ & $m / 1+ \pm q / 41$ & يى ئيرى & \\
\hline$<. / . .1$ & .1 .09 & $<+/ * 1$ & $<\cdot 1 \cdot+1$ & $<.1 . .1$ & $<+1 \cdot .1$ & & $11 / F \Delta \pm / \Delta A$ & $11 / Y \Delta \pm \cdot / \Delta Y$ & قبل & \\
\hline \multirow[t]{2}{*}{$t=11 / .}$. & $\mathrm{t}=\mathrm{V} / \mathrm{rA}$ & $\mathrm{t}=1 t / q \varepsilon$ & $t=|Q / A|$ & $t=r p / r q$ & $t=|v / 1|$ & $<+1 .+1$ & $|\Psi /| \pm \cdot / M A$ & WI/RAt/AY & بعد & \\
\hline & & & & & & & $\mid f / r \Psi \pm 1 / .8$ & 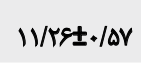 & يهكيرى & \\
\hline$<* 1.01$ & $<.1 .+1$ & $<+1 . .1$ & $<.1 .+1$ & $<.1 . .1$ & $<.1 .+1$ & & $\Delta / \Delta / \pm+|8|$ & $\Delta / q) \pm \cdot / N$ & قبل & \multirow{3}{*}{ أستأه تحمل سابي } \\
\hline \multirow[t]{2}{*}{$t=11 / v a$} & $t=V / F r$ & $t=I V / v a$ & $\mathrm{t}=1 F / \mathrm{V}$ & $\mathrm{t}=r+/ r q$ & $\mathrm{t}=|g / \pi|$ & $<+1 .+1$ & $V / / \mp \pm \pm / \Delta q$ & $\Delta / \mathscr{R} \pm \cdot N$ & بعل & \\
\hline & & & & & & & $N \cdot V \pm \cdot M$ & $\Delta / f \mid \pm \cdot M$ & يقىيرى & \\
\hline$<+1 . .+1$ & $<\cdot 1 . .1$ & $<+1 . .1$ & $<* / .+1$ & $<\cdot 1+\infty 1$ & $<+1 .+1$ & & $9 / \Delta . \pm . / A V$ & $g / M \pm \pm / W$ & قبل & \multirow{3}{*}{ استرنوكلوئيدوماستوتئيد } \\
\hline \multirow[t]{2}{*}{$t=q / A Y$} & $\mathrm{t}=s / \pi r$ & $\mathrm{t}=\mid \xi / 1$ & $t=1 Q / \Delta A$ & $t=r V / T r$ & $t=N / P F$ & $<.1 . .1$ & VIAYI./ES & $E / F \cdot \pm \cdot N E$ & بعل & \\
\hline & & & & & & & $N 8+ \pm+/ N 8$ & $g / r+ \pm \cdot M$ & يقى يرى & \\
\hline
\end{tabular}

توانبخننى

* P-value for independent t-test

** P-value for paired t-test in treatment group

$* * * \mathrm{P}$-value for paired t-test in control group

**** $\mathrm{P}$-value for repeated measurement

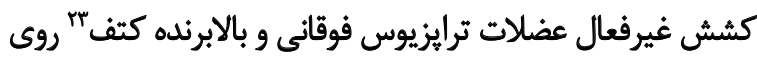

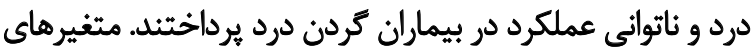

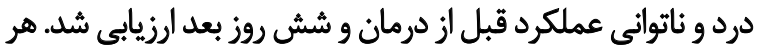

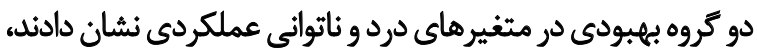

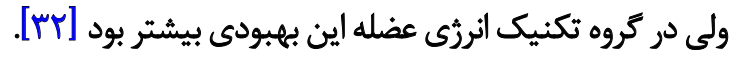

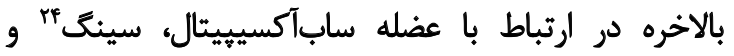

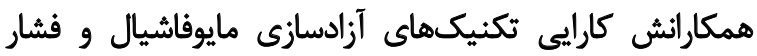

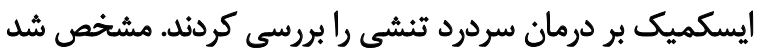

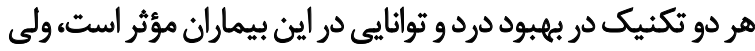

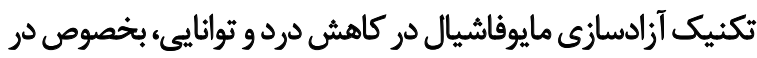

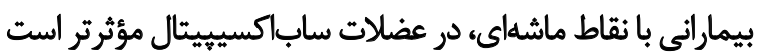

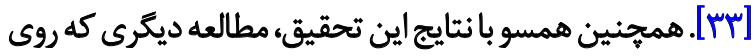

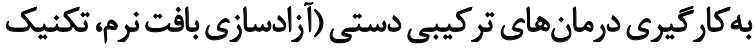

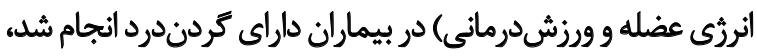

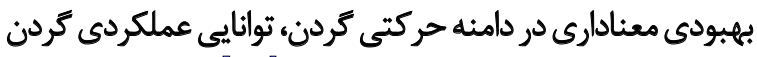

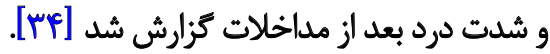

كنترل، باعث بهبود فركانس، شدت و مدت زمان سردرد و آستانه

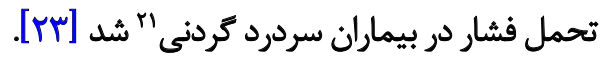
از طرف ديكر در ارتباط با عضله ترايزيوس فوقانى، ضيايىفر و

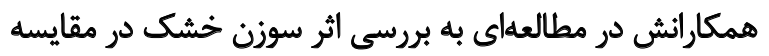

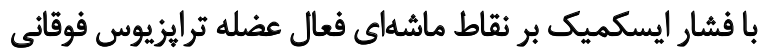

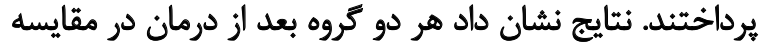

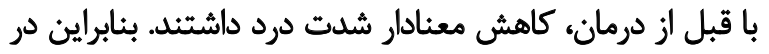

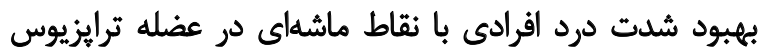

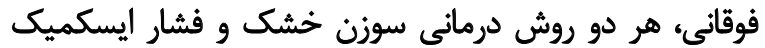

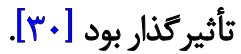

همجنين طباطبايى و همكارانش با مقايسه دو روش فشار و رها

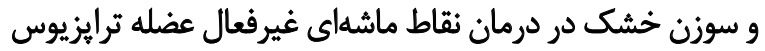

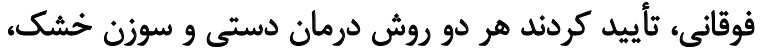

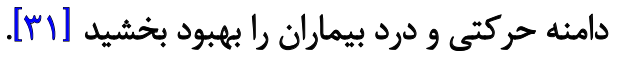
فادكه و همكارانش به مقايسه تأثير تكنيك انرزى عضله بr و و 
است. مجموعه ترايجمنو- سرويكال محدودهاى است كه توسط

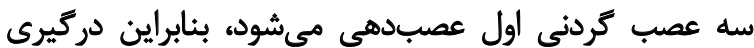

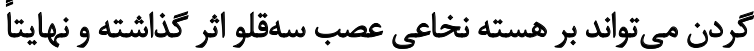

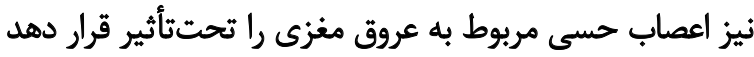

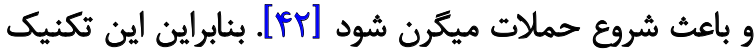

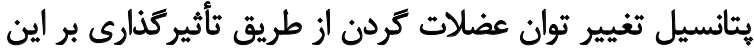

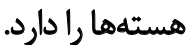

r. يك ارتباط آناتوميكى بين عضلات اطراف مهرههاى كردن بران

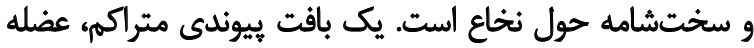

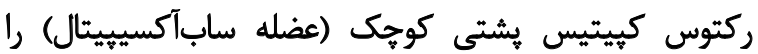

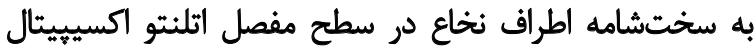

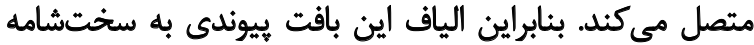

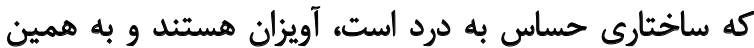

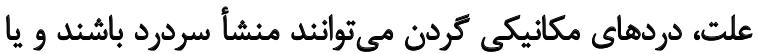

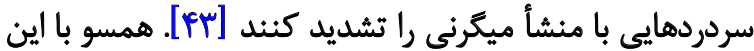

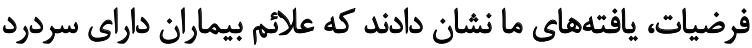
ميكرنى، بعد از درمان نقاط ماشهاي عضلات كردن كاهش باني بيدا كرد.

\section{تئيجليرى}

براساس يافتههاي مطالعه حاضر، تكنيك آزادسازى عضلات

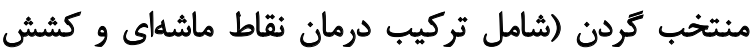

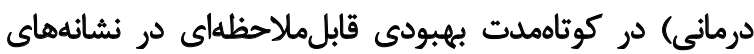

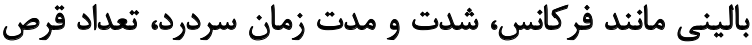

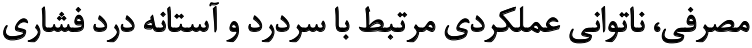

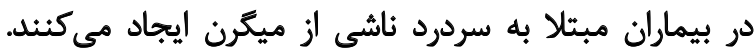

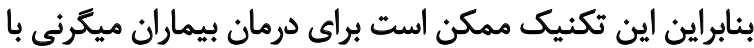
نقاط ماشهاي در عضلات كردن استفاده شود. يكى از محدوديثهاى اين يُروهش، مدت زمان كوتاه درمان

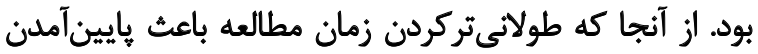

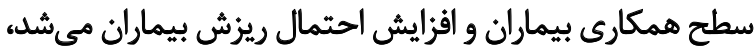

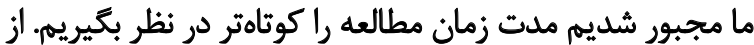

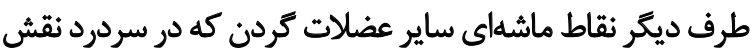

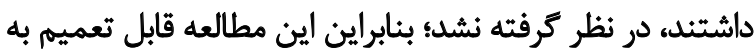

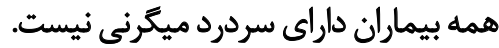

در طى اين جلسات، براى بيماران آموزش هاى لازم براى اصلاح

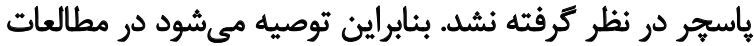

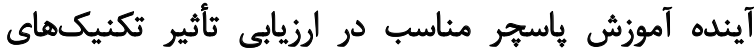

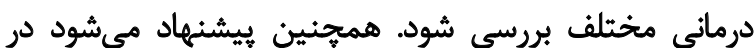

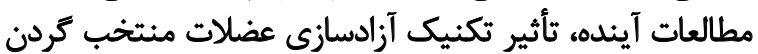

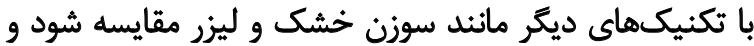

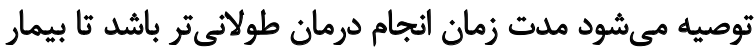

$$
\text { بيشتر تحتنظر باشد. }
$$

همجنين رمضانى و همكارانش مطالعهاي براي بررسى تأثير

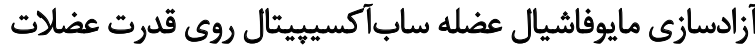

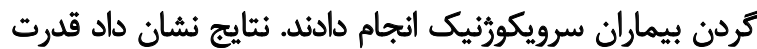

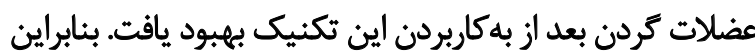

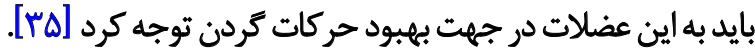
تئورىهاى مختلفى در ارتباط با آسيبشناسى نقاط ماشهاى

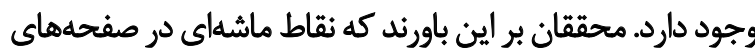

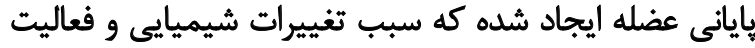

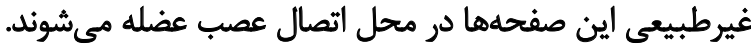

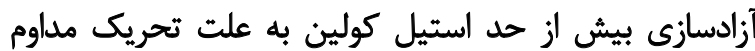

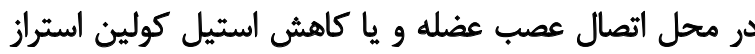
سبب تشكيل باندهاى سفت مى شونداند در اين بائ باندهاى سفت

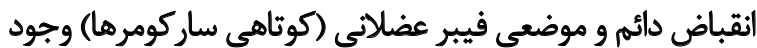

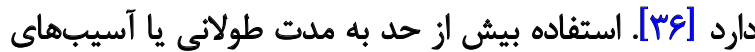

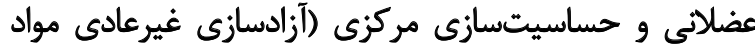

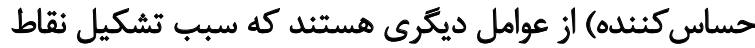

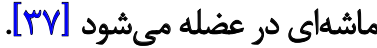

تكنيك آزادسازى بافت نرم، به تغييرات تردش خون و افزايش

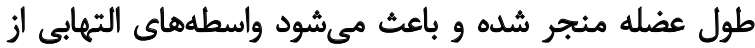

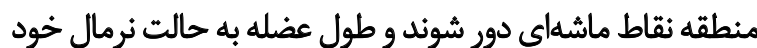

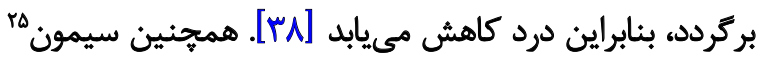

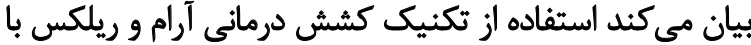

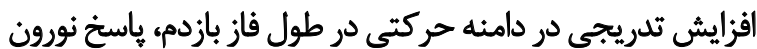

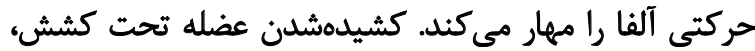

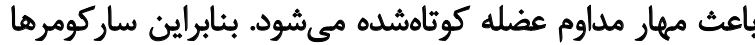

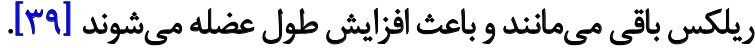

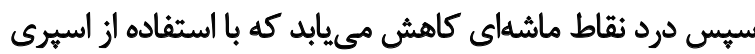

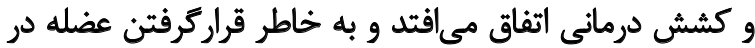

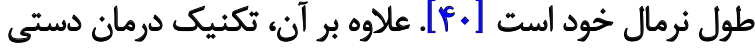

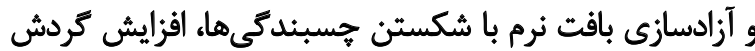

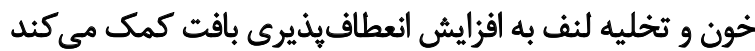

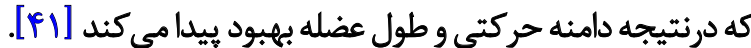
اين يثوهش بيشنهاد مى كند بعد از درمان نقاط ماشهاى براى

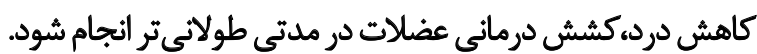

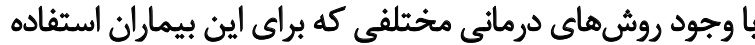

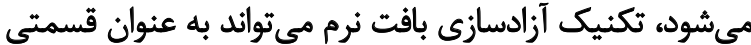

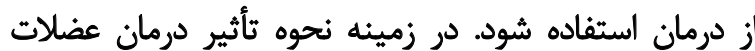

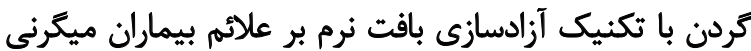

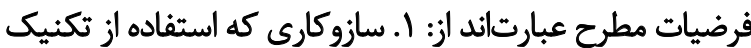

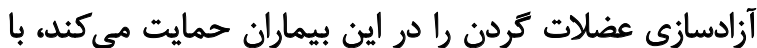

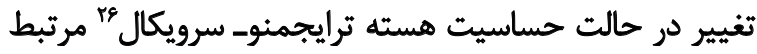




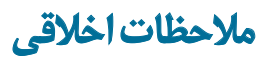

بيزوى ائ اصول اخلاق يثوهش

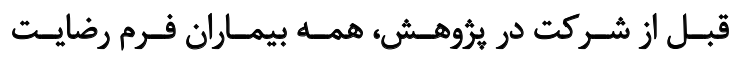

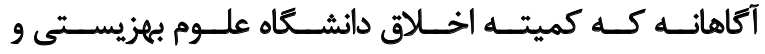

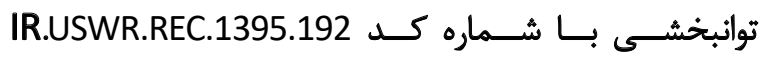

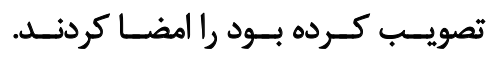

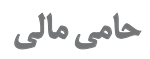

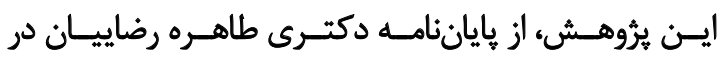

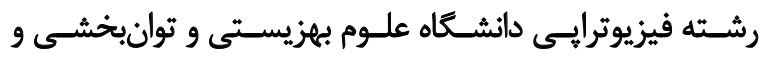

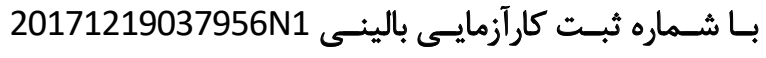
اسـتخراج شــده اسـت كارازمايست مشار كت نويسئد مكان

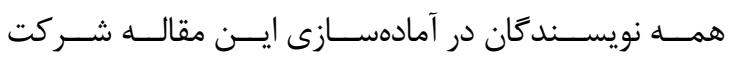
داشــتهاند. تقارض مناقع

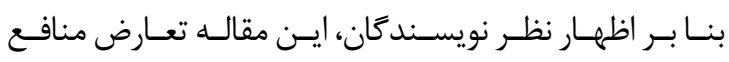

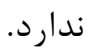




\section{References}

[1] Stovner L, Hagen K, Jensen R, Katsarava Z, Lipton R, Scher A, et al. The global burden of headache: $A$ documentation of headache prevalence and disability worldwide. Cephalalgia. 2007; 27(3):193210. [DOI:10.1111/j.1468-2982.2007.01288.x] [PMID]

[2] Levin M. The International Classification of Headache Disorders, (ICHD III)-changes and challenges. Headache. 2013; 53(8):138395. [DOI:10.1111/head.12189]

[3] Headache Classification Committee of the International Headache Society (IHS). The international classification of headache disorders. Cephalalgia. 2013; 33(9):629-808. [DOI:10.1177/0333102413485658] [PMID]

[4] Tepper SJ, Tepper DE. Diagnosis of migraine and tension-type headaches. In SJ Tepper, DE Tepper, editors. The Cleveland Clinic Manual of Headache Therapy. Berlin: Springer; 2014. [DOI:10.1007/978-3-319-04072-1_1]

[5] Amin FM, Asghar MS, Hougaard A, Hansen AE, Larsen VA, de Koning PJ, et al. Magnetic resonance angiography of intracranial and extracranial arteries in patients with spontaneous migraine without aura: A cross-sectional study. The Lancet Neurology. 2013; 12(5):454-61. [DOI:10.1016/S1474-4422(13)70067-X]

[6] Olesen J, Burstein R, Ashina M, Tfelt-Hansen P. Origin of pain in migraine: Evidence for peripheral sensitisation. The Lancet Neurology. 2009; 8(7):679-90. [DOI:10.1016/S14744422(09)70090-0]

[7] Calandre E, Hidalgo J, García-Leiva J, Rico-Villademoros F. Trigger point evaluation in migraine patients: An indication of peripheral sensitization linked to migraine predisposition? European Journal of Neurology. 2006; 13(3):244-9. [DOI:10.1111/ j.1468-1331.2006.01181.x] [PMID]

[8] Tali D, Menahem I, Vered E, Kalichman L. Upper cervical mobility, posture and myofascial trigger points in subjects with episodic migraine: Case-control study. Journal of Bodywork and Movement Therapies. 2014; 18(4):569-75. [DOI:10.1016/j. jbmt.2014.01.006] [PMID]

[9] Fernández-de-Las-Peñas C, Cuadrado M, Pareja J. Myofascial trigger points, neck mobility and forward head posture in unilateral migraine. Cephalalgia. 2006; 26(9):1061-70. [DOI:10.1111/j.1468-2982.2006.01162.x] [PMID]

[10] Ferracini GN, Chaves TC, Dach F, Bevilaqua-Grossi D, Fernández-de-las-Peñas C, Speciali JG. Relationship between active trigger points and head/neck posture in patients with migraine. American Journal of Physical Medicine \& Rehabilitation. 2016; 95(11):831-9. [DOI:10.1097/PHM.0000000000000510] [PMID]

[11] Chaibi A, Tuchin PJ, Russell MB. Manual therapies for migraine: A systematic review. The Journal of Headache and Pain. 2011; 12(2):127-33. [DOI:10.1007/s10194-011-0296-6] [PMID] [PMCID]

[12] Luedtke K, Allers A, Schulte LH, May A. Efficacy of interventions used by physiotherapists for patients with headache and migraine-systematic review and meta-analysis. CephalaIgia. 2016; 36(5):474-92. [DOI:10.1177/0333102415597889] [PMID]
[13] John P, Sharma N, Sharma CM, Kankane A. Effectiveness of yoga therapy in the treatment of migraine without aura: $A$ randomized controlled trial. Headache. 2007; 47(5):654-61. [DOI:10.1111/j.1526-4610.2007.00789.x] [PMID]

[14] Noudeh YJ, Vatankhah N, Baradaran HR. Reduction of current migraine headache pain following neck massage and spinal manipulation. International Journal of Therapeutic Massage \& Bodywork. 2012; 5(1):5-13. [DOI:10.3822/ijtmb. v5i1.115]

[15] Ghanbari A, Askarzadeh S, Petramfar P, Mohamadi M. Migraine responds better to a combination of medical therapy and trigger point management than routine medical therapy alone. Neurorehabilitation. 2015; 37(1):157-63. [DOI:10.3233/NRE-151248] [PMID]

[16] Chaitow L, Delany J. Clinical application of neuromuscular techniques: The upper body, Shoulder, arm and hand. London: Elsevier; 2008.

[17] Couch JR, Group AVPS. Amitriptyline in the prophylac tic treatment of migraine and chronic daily headache. Headache. 2011; 51(1):33-51. [DOI:10.1111/j.1526 4610.2010.01800.x] [PMID]

[18] Mohammadi N, Sajadinejad MS, Taghavi MR, Ashjazadeh N. Effects of cognitive-behavioral group therapy on recurrent headaches in Iranian culture. Psychological Reports. 2008, 103(3):893-8. [DOI:10.2466/pr0.103.3.893-898] [PMID]

[19] Reeves JL, Jaeger B, Graff-Radford SB. Reliability of the pressure Algometer as a measure of myofascial trigger point sensitivity. Pain. 1986; 24(3):313-21. [DOI:10.1016/03043959(86)90117-X]

[20] DeStefano LA. Greenman's principles of manual medicine. Philadelphia: Lippincott Williams \& Wilkins; 2011.

[21] Hamilton L, Boswell C, Fryer G. The effects of high-velocity, low-amplitude manipulation and muscle energy technique on suboccipital tenderness. International Journal of Osteopathic Medicine. 2007; 10(2-3):42-9. [DOI:10.1016/j. ijosm.2007.08.002]

[22] Jafari M, Bahrpeyma F, Togha M. Effect of ischemic compression for cervicogenic headache and elastic behavior of active trigger point in the sternocleidomastoid muscle using ultrasound imaging. Journal of Bodywork and Movement Therapies. 2017; 21(4):933-9. [DOI:10.1016/j. jbmt.2017.01.001] [PMID]

[23] Espí-López GV, Ruescas-Nicolau MA, Nova-Redondo C Benítez-Martínez JC, Dugailly PM, Falla D. Effect of soft tissue techniques on headache impact, disability, and quality of life in migraine sufferers: A pilot study. The Journal of Alternative and Complementary Medicine. 2018; 24(11):1099-107. [DOI:10.1089/acm.2018.0048] [PMID]

[24] Bevilaqua-Grossi D, Gonçalves MC, Carvalho GF, Florencio LL, Dach F, Speciali JG, et al. Additional effects of a physical therapy protocol on headache frequency, pressure pain threshold, and improvement perception in patients with migraine and associated neck pain: A randomized controlled trial. Archives of Physical Medicine and Rehabilitation. 2016; 97(6):866-74. [DOI:10.1016/j.apmr.2015.12.006] [PMID] 
[25] Ferragut-Garcías A, Plaza-Manzano G, Rodríguez-Blanco C, Velasco-Roldán O, Pecos-Martín D, Oliva-Pascual-Vaca J, et al. Effectiveness of a treatment involving soft tissue techniques and/or neural mobilization techniques in the management of tension-type headache: A randomized controlled trial. Archives of Physical Medicine and Rehabilitation. 2017; 98(2):211-9. e2. [DOI:10.1016/j.apmr.2016.08.466] [PMID]

[26] Szikszay T. Effectiveness of a treatment involving soft tissue techniques and/or neural mobilization techniques in the management of tension-type headache: $A$ randomized controlled trial. Physioscience. 2017; 13(04):186-7. [DOI:10.1055/s-0035-1567230]

[27] Hanten WP, Olson SL, Butts NL, Nowicki AL. Effectiveness of a home program of ischemic pressure followed by sustained stretch for treatment of myofascial trigger points. Physical Therapy. 2000; 80(10):997-1003. [PMID]

[28] Ghanbari A, Rahimijaberi A, Mohamadi M, Abbasi L, Sarvestani FK. The effect of trigger point management by positional release therapy on tension type headache. Neurorehabilitation. 2012; 30(4):333-9. [DOI:10.3233/NRE-2012-0764]

[29] Cerritelli F, Ginevri L, Messi G, Caprari E, Di Vincenzo M, Renzetti $C$, et al. Clinical effectiveness of osteopathic treatment in chronic migraine: 3-Armed randomized controlled trial. Complementary Therapies in Medicine. 2015; 23(2):149-56. [DOI:10.1016/j.ctim.2015.01.011] [PMID]

[30] Ziaeifar M, A'rab AM, Karimi N, Mosalla-Nezhad Z. The effect of dry needling compared with ischemic pressure on pain intensity on active trigger point in upper trapezius muscle. Archives of Rehabilitation. 2013; 14(2):86-92. [DOI: 10.1016/j. jbmt.2013.11.004] [PMID]

[31] Tabatabaiee A, Ebrahimi I, Ahmadi A, Sarrafzadeh J. [Comparison between the effect of pressure release and dry needling on the treatment of latent trigger point of upper trapezius muscle (Persian)]. Physical Treatments-Specific Physical Therapy Journal. 2013; 3(3):9-15.

[32] Phadke A, Bedekar N, Shyam A, Sancheti P. Effect of muscle energy technique and static stretching on pain and functional disability in patients with mechanical neck pain: A randomized controlled trial. Hong Kong Physiotherapy Journal. 2016; 35:511. [DOI:10.1016/j.hkpj.2015.12.002] [PMID] [PMCID]

[33] Singh LR, Chauhan V. Comparison of efficacy of myofascial release and positional release therapy in tension type headache. Journal of Medical Science and clinical Research. 2014; 2(9):2372-9.

[34] Ghodrati M, Mosallanezhad Z, Shati M, Rastgar Koutenaei F, Nourbakhsh MR, Noroozi M. The effect of combination therapy; manual therapy and exercise, in patients with nonspecific chronic neck pain: A randomized clinical trial. Physical Treatments-Specific Physical Therapy Journal. 2017; 7(2):11321. [DOI:10.32598/ptj.7.2.113]

[35] Ramezani E, Arab AM. The effect of suboccipital myofascial release technique on cervical muscle strength of patients with cervicogenic headache. Physical Treatments-Specific Physical Therapy Journal. 2017; 7(1):19-28. [DOI:10.29252/nrip. ptj.7.1.19]
[36] Mense S. Nociception from skeletal muscle in relation to clinical muscle pain. Pain. 1993; 54(3):241-89. [DOI:10.1016/03043959(93)90027-M]

[37] Simons DG. New aspects of myofascial trigger points: etiological and clinical. Journal of Musculoskeletal Pain. 2004; 12(34):15-21. [DOI:10.1300/J094v12n03_03]

[38] Ajimsha M. Effectiveness of direct vs indirect technique myofascial release in the management of tension-type headache. Journal of Bodywork and Movement Therapies. 2011; 15(4):431-5. [DOI:10.1016/j.jbmt.2011.01.021] [PMID]

[39] Simons DG. Understanding effective treatments of myofascial trigger points. Journal of Bodywork and Movement Therapies. 2002; 6(2):81-8. [DOI:10.1054/jbmt.2002.0271]

[40] Travell JG, Simons DG. Myofascial pain and dysfunction: The trigger point manual. Philadelphia: Lippincott Williams \& Wilkins; 1983.

[41] Saratchandran R, Desai S. Myofascial release as an adjunct to conventional occupational therapy in mechanical low back pain. Indian Journal of Occupational Therapy. 2013; 45:3-7.

[42] Goadsby PJ, Classey JD. Glutamatergic transmission in the trigeminal nucleus assessed with local blood flow. Brain Research. 2000; 875(1-2):119-24. [DOI:10.1016/S00068993(00)02630-5]

[43] Palomeque-del-Cerro L, Arráez-Aybar LA, Rodríguez-Blanco C, Guzmán-García R, Menendez-Aparicio M, Oliva-Pascual-Vaca Á. A systematic review of the soft-tissue connections between neck muscles and dura mater: The myodural bridge. Spine. 2017; 42(1):49-54. [DOI:10.1097/BRS.0000000000001655] [PMID] 